\title{
Management of Climatic Heat Stress Risk in Construction: A review of practices, methodologies, and future research
}

\author{
Steve Rowlinson ${ }^{1}$, Andrea Yunyan $\mathrm{Jia}^{1}{ }^{1}$, Baizhan $\mathrm{Li}^{2}$, Carrie Chuanjing $\mathrm{Ju}^{1}$
}

\begin{abstract}
Climatic heat stress leads to accidents on construction sites brought about by a range of human factors emanating from heat induced illness, and fatigue leading to impaired capability, physical and mental. Heat stress is an occupational hazard in construction work. The authors take the approach of re-engineering the whole safety management system rather than merely focusing on incremental improvement. Technically, climatic heat stress is determined by six key factors: air temperature, humidity, radiant heat, and wind speed (the environment), metabolic heat generated by physical activities, and the "clothing effect" that moderates the heat exchange between the body and the environment. From a systems point of view, the task of risk management is to identify boundaries of safety and create opportunities to develop coping skills at these boundaries. The boundaries of safety to be identified in climatic heat stress risk management are basically environmental thresholds that define safe work limits under certain work-rest regimens. By making use of existing heat stress indices and heat stress management processes, heat stress risk on construction sites can be managed in three ways: (1) control of environmental heat stress exposure through use of an action-triggering threshold system, (2) control of Continuous Work Time (CWT, referred by Maximum Allowable Exposure Duration) with mandatory work-rest regimens, and (3) enabling selfpaced working through empowerment of employees. Informed by systems thinking approach, existing heat stress practices and methodologies are critically reviewed and the authors propose a systemic methodology for an action-triggering, localised, simplified threshold system to facilitate effective decisions by frontline supervisors. The authors point out the need for "regional based" heat stress management practices that reflect unique climatic conditions, working practices and acclimatisation propensity by local workers indifferent geographic regions. The authors set out the case for regional, rather than international, standards that account for this uniqueness and which are derived from site-based rather than laboratory-based research.
\end{abstract}

[Key Words] Climatic heat stress, construction accidents, maximum allowable exposure duration, Continuous Work Time, recovery time, self-paced working

\section{Introduction}

Increasing ambient temperature is one of the potential occupational hazards associated with climate change (IPCC, 2007; Schulte \& Chun, 2009). How working people are affected by, whilst adapting to, climatic heat is becoming an important issue in the global research agenda (Kjellstrom et al., 2009a; Lundgren et al., 2013). Among the identified vulnerable occupational populations, construction workers are a top priority due to the often informal manner in which the construction industry organizes itself and its importance to developed and developing economies (Gillen \& Gittleman, 2010; Lin \& Chan, 2009). Theories and measurement of interaction between the human body and environmental heat have been explored and developed for more than two centuries in the laboratory (Blagden, 1775a, 1775b), independent from the social and organizational context of the occupational setting. Heat stress was studied for military management purposes during WWII

\footnotetext{
${ }^{1}$ Department of Real Estate and Construction, The University of Hong Kong, Hong Kong

2 Faculty of Urban Construction and Environmental Engineering, Chongqing University, China
} 
(e.g.Yaglou \& Minard, 1956) and later in the mining industry because of its importance to industrial development (Lind et al., 1957). The construction industry did not recognize it as a risk factor in accidents until quite recently (CSAO, 2000; Frimpong et al., 2003) although seasonal variation in construction accident rate with a peak appearing in summer months had been observed since at least the late 1970s (Helander, 1980). Systematic research on its relationship with accidents and accident prevention has developed slowly in many regions. The aim of this paper is to review current climatic heat risk management methodologies and practices in order to identify the key factors and research objectives in developing appropriate strategies to combat heat stress and, informed by a systems thinking approach, to formulate action-triggering, localized, industry specific guidelines for heat stress management in the construction industry, which can act as a driver to re-engineer the safety management system as a whole.

\section{Climatic heat risk and construction accidents}

Construction work is characterized as heavy physical work that subjects workers to physical strain as well as inducing metabolic heat gain. In a survey conducted by the authors in 2011, 17 of 37 trades reported cases of heat-induced illness on construction sites. $17 \%$ of workers reported experiences of heat-induced illness ${ }^{1}$. Over-exposure to heat can induce disorders such as heat rash, heat cramps, heat syncope or fainting, heat exhaustion, or heat stroke. A risk originating from our natural environment, heat stroke as a direct consequence of heat stress can be fatal. For example, Chan (2012) examined Hong Kong newspapers during 2007 to 2011 and found 43 heat stress-related accidents in construction sites, including 11 fatalities. Consequences of heat stress lead to managerial risk related to productivity, cost and low worker morale, and legal risk for the organization from subsequent accidents (Edwards \& Bowen, 1998).

Except for cases of heat illness happening on site, heat stress tends to induce other construction accidents through physical fatigue, impaired mental capacity, and misuse of inconvenient Personal Protective Equipment (PPE). An early study with electrical journeymen found the rise of environmental temperature is directly associated with the level of fatigue (NECA, 1974, summarized in Koehn \& Brown 1985) which is shown to be one of the most important factors leading to construction accidents (Chan, 2011; Garrett \& Teizer, 2009). Heat cramps and fainting are dangerous for both the workers and their co-workers exposed to other hazards on site. A significant drop in mental performance is observed at temperatures above $32.2^{\circ} \mathrm{C}$ (Basic Effective Temperature) in a hot-humid environment and $33^{\circ} \mathrm{C}$ in a hot-dry environment (Sharma et al., 1983). The reduced performance includes speed of response, reasoning ability, visual perception, associative learning, and mental alertness which has been reported to be one of the causations of fatal accidents (e.g. Chia et al., 2005).

The most commonly used Personal Protective Equipment (PPE) on construction sites, i.e. safety helmets, reflective vests, and safety boots, often increase workers' heat strain. The materials used in reflective vests are often made of water impermeable materials that block effective heat dissipation and leads to workers' reluctance to wear them. Safety helmets, especially those without ventilation, can lift the temperature inside the helmet dramatically. For example, the authors recorded an air temperature of $57^{\circ} \mathrm{C}$ inside a worker's safety helmet in August 2011 when the environmental temperature was $33^{\circ} \mathrm{C}^{1}$. In such circumstances workers will naturally take off their helmets from time to time to alleviate heat stress and so expose themselves to other hazards on site. Similar problem exists with eye protection equipment. Choudhry and Fang (2008) found an eye injury accident was caused by worker's reluctance to wear protective glasses in hot weather because, as self-reported by the worker, "it is harsh to wear glasses under heat or sunlight and it is even difficult to see with grubby or mucky glasses"(p.578).

Pattern of climatic heat risk varies with types of construction sites as well as stages of a project lifecycle. For example, sites on civil engineering work such as road construction are more vulnerable 
to radiant heat as determined by the open site characteristics. Building sites, on the other hand, naturally have more shaded areas on site but suffer more on heat stress generated by high humidity and lack of ventilation, depends on the specific location workers are working on. Indoor work such as HAVC installation at the stage after glassing is similar to works in a confined space. While tunneling work suffers from high humidity and lack of fresh air which add to the heat stress in summer. Heat stress falling on different characteristics of sites generates diverse issues on risk mitigation measures. For example, the large size of civil engineering site aggravates travel difficulties in hot weather in developing countries which influence supervision (Abdelsalam \& Gad, 2009). Whilst in sites of maintenance and alteration projects, workers often work alone thus makes communication difficult, buddy caring impossible and self-awareness more important.

The above illustrates that climatic heat in construction sites is not an isolated risk that can be effectively managed through an incremental improvement to the existing project management system. Construction safety management systems are developed assuming a steady state and condition and rarely take the changing thermal environment into consideration. From a systems point of view, the task of risk management is to identify boundaries of safety and create opportunities to develop coping skills at these boundaries (Rasmussen, 1997). The boundaries of safety to be identified in climatic heat risk management are basically environmental thresholds that define safe work limit under certain work-rest regimens. The following reviews factors and methods for identification of boundary conditions for managing heat stress risks.

\section{Identification of safe boundaries: key factors and measures of heat stress}

Technically, the objective of climatic heat risk management is to monitor and control the impact of heat stress on heat strain in order to protect workers from heat induced illness. Whereas heat stress is the heat load imposed on the human body, including environmental heat, metabolic heat and the thermal effect of clothing; heat strain is the physiological response of the human body in dissipating excess heat (ACGIH, 2009; Malchaire et al., 2001).

\subsection{Indicators of heat strain}

As the ultimate objective is to control heat strain under a tolerable physiological limit, the job of heat stress management can be done once-for-all by individualised monitoring of physiological parameters and controlling them to a tolerable limit. Indicators for heat strain are identified as body core temperature, skin temperature, heart rate and the loss of body mass through sweating (ISO 9886, 2004). ISO 7933 (2004) sets $38^{\circ} \mathrm{C}$ as the upper limit of body core temperature as recommended by WHO (1969). The upper limit of heart rate as recommended by the WHO is 110 beats per minute (bpm). More specifically, ACGIH (2009) suggests a limit of $120 \mathrm{bpm}$ for one-minute recovery heart rate. Alternatively, and more logically as it takes age into account, the UK Health and Safety Executive (HSE) (2012a) suggests the workplace heart rate threshold be calculated as Age multiplied by 0.65 subtracted from 165. For reference limits in body mass loss, the ISO 7933 (2004) set $7.5 \%$ body mass loss as the upper limit for an average person and $5 \%$ for $95 \%$ of the working population. Lu and Zhu (2007) observed a sweat loss of 1-3\% of body weight as the upper limit of heat tolerance in their Chinese sample.

A number of devices, e.g. the Polar ${ }^{\mathrm{TM}}$ Heart Rate Monitor (Miller et al., 2011) and the CorTemp Ingestible Core Body Thermometer Pill (NASA, 2006), have enabled direct monitoring and control of heat strain. However, direct intervention on heat strain at the workplace raises safety issues from its intrusive measurement approach and is constrained by cost and its disruption to work. So far these devices are used for research only and are not considered to provide practical methods for monitoring and controlling heat risks in a systemic way.

Inconvenience in direct control of physiological strain directs research focus to the control of heat risk itself. By the 1980s heat strain was commonly agreed to be predicted by six heat stress factors 
including (1) air temperature, (2) humidity, (3) solar radiant heat and (4) wind speed, i.e. environmental heat, (5) metabolic heat, generated by physical activities, that varies with work pace and accumulates with work time, and (6) the clothing effect as a moderator of heat dissipation (Parsons, 1995). Thus, risk can be mitigated through control of three manageable factors: environmental heat stress, work pace and continuous work time. The following sections review major tools and methods in identifying safe boundaries, in this case setting environmental thresholds in the work setting.

\subsection{Environmental heat stress indices and environmental thresholds}

\subsubsection{The WBGT index}

A heat stress index provides a scale to measure the "hotness" of the environment based on human perception. The most widely used heat stress index is the Wet Bulb Globe Temperature (WBGT) that encapsulates air temperature, humidity, radiant heat and wind speed in a single index. The WBGT index is based on the simple idea of signaling "how hot it is" through a device that mimics a sweating human body receiving heat from air temperature and solar radiation while cooled by wind (Budd, 2008; Yaglou \& Minard, 1956). The index was an outcome of progressive improvement on the Effective Temperature index originally devised in 1923 (Bedford, 1946; Gagge et al., 1970; Houghten \& Yagloglou, 1923; Rohles, 1973; Yaglou, 1950; Yaglou \& Minard, 1957). The instrument measuring WBGT is composed of a shielded dry bulb thermometer, a natural wet bulb thermometer and a globe thermometer - a black globe heated by solar radiation (Budd, 2008; Hatch, 1973). The WBGT index is a weighted average of the three readings (cf. ISO 7243, 1989). An evaluation of its face validity can be found in Parsons (2006). It is a convenient measurement of environmental heat stress that can be easily implemented for heat stress management in an industrial environment by laymen.

Despite recent discussion of its limitations in underestimating the effect of wind speed (Miller \& Bates, 2007) and its inability to measure the effect of the other two important heat stress factors, metabolic rate and clothing effect (Parsons, 2006), the WBGT remains a valid heat index for managing occupational heat stress in a convenient procedure. It underlines two most widely used environmental threshold systems, the annually updated Threshold Limit Values (TLVs ${ }^{\circledR}$ ) by the American Conference of Governmental and Industrial Hygienists (ACGIH, 2013) and the Reference Values of ISO 7243 (1989), which underpin the occupational safety and health guidelines of the USA (NIOSH, 1972, 1986; OSHA, 1999a), Canada (Ontario Ministry of Labour, 2013; Saskatchewan Ministry of Labour and Workplace Safety, 2000; Work Safe Alberta, 2009), P. R. China(GB/T 4200, 2008; GBZ 2.2, 2007; GBZ/T 189.7, 2007; GBZ/T 229.3, 2010), Republic of China (IOSH, 1997), UK (BS EN 27243, 1994; HSE, 2012b), Australia (AIOH, 2003), New Zealand (OSHS, 1997), etc. Supplementary methods have been devised to address the above mentioned limitations by standardising wind speed, categorising metabolic rate by types of work, and setting up clothing adjustment values for major work uniforms (Ashley et al., 2008; Bernard, 1999).

\subsubsection{Other heat stress indices in use}

Two other widely used heat indices are Humidex (Masterton \& Richardson, 1979), a simplified version of Belding and Hatch's (1955) Heat Stress Index, in Canada and Heat Index (Steadman, 1979, 1984 ) in the US through public weather reports. Both indices integrate the effects of air temperature and humidity and can be obtained through a thermometer and a humidity meter, thus further easing the measurement process with a much lower cost. They enable employers to make use of publicly available information, i.e. temperature and humidity reports from the local observatory, thus cutting down the cost for resources spent on staffing and equipment needed for WBGT surveillance. However, the fact that workplace micro-climate often deviates significantly from the general climate of a city or district has put their validity for workplace risk assessment in doubt. The intrinsic problem in the development of these two indices is that they ignore the influence of solar radiation and wind speed that play important roles in heat stress experienced by outdoor workers. Despite 
these concerns they are often recommended as alternative indices on occasion when WBGT is not available (e.g. CSAO, 2009; CSAO, 2010).

Specific to the working environment of air crews, the US Airforce simplified the WBGT index into the Fighter Index of Thermal Stress (FITS) which can be interpreted from reading a simple chart of air temperature and relative humidity (Stribley \& Nunneley, 1978), assuming typical metabolic rates and standard clothing of pilot in a cockpit. It was adopted in the US Environmental Protection Agency's (EPA) heat stress management guidelines for agriculture work (EPA, 1993). However the FITS used in an industrial setting has to be accompanied by many more adjustment factors such as radiation, wind speed, etc., which complicates the simplified procedure and impairs its convenience and validity. This indicates a need to develop occupation specific guidelines which address workplace characteristics as well as culture and norms of a specific crew of workers.

A recently developed index, the Thermal Work Limit (TWL), tries to improve on the use of WBGT devised thresholds by making better estimation of the effect of air cooling power. The TWL was developed from the 1989 version of ISO 7933, the Required Sweat Rate (RSR) model (ISO 7933, 1989). Following the rationale of the RSR model that indicates environmental heat stress through personal physiological strain, the TWL model encapsulates the environmental parameters into a single index by converting them into the equivalent metabolic rate needed to maintain the body's thermal balance (Brake, 2002; Brake \& Bates, 2002). The TWL model is used as an alternative to heat indices in guidelines for heat stress management in the Australian mining industry (Bell, 2012; Corleto, 2011; Taylor \& O'Sullivan, 2012) and is specified as a tool to set up thresholds for workplace heat stress control in Abu Dhabi's industrial guidelines (Abu Dhabi EHS Center, 2012). As the TWL is developed based on a slightly different set of assumptions to the ISOs, further evidence of its validation is yet to be reported.

\subsection{Metabolic heat and work pace}

There are three existing methods for assessing metabolic rate in the heat stress management guidelines: by classification, by continuum, and by using representative values. In guidelines by ACGIH and ISO 7243 workload is classified into four categories, i.e. light $\left(>65, \leq 130 \mathrm{~W} / \mathrm{m}^{2}\right)$, moderate $\left(>130, \leq 200 \mathrm{~W} / \mathrm{m}^{2}\right)$, heavy $\left(>200, \leq 260 \mathrm{~W} / \mathrm{m}^{2}\right)$, and very heavy $\left(>260 \mathrm{~W} / \mathrm{m}^{2}\right)$. In developing the threshold limit values for each category, the mid-point of each range is used as a representative value, i.e. light $\left(100 \mathrm{~W} / \mathrm{m}^{2}\right)$, moderate $\left(165 \mathrm{~W} / \mathrm{m}^{2}\right)$, heavy $\left(220 \mathrm{~W} / \mathrm{m}^{2}\right)$, very heavy $\left(290 \mathrm{~W} / \mathrm{m}^{2}\right)$. The thresholds developed based on these representative values are thus reduced to as few as four values. For example, on the assumed condition of "sensible air movement", the thresholds given by ISO 7243 are $30^{\circ} \mathrm{C}$-WBGT for light work, $28^{\circ} \mathrm{C}$-WBGT for moderate work, $26^{\circ} \mathrm{C}$-WBGT for heavy work and $25^{\circ} \mathrm{C}-\mathrm{WBGT}$ for very heavy work. Whilst easy to understand and implement, there are often conditions at the workplace such that actual metabolic rate of workers is close to the margins of the light, moderate or heavy work categories (Parsons, 2006, p. 373). To address this issue both guidelines provide plotted curves establishing the continuous association between WBGT and metabolic rate (ACGIH, 2013, p. 208; ISO 7243, 1989, p. 10).

The metabolic heat generated by physical activities is closely related to work pace. In the simplified thresholds system, work pace is categorized into four ranges, indicated by the percentage of work in a work-recovery cycle, i.e. $75-100 \%, 50-75 \%, 25-50 \%, 0-25 \%$, corresponding to thresholds in 0.5 to $1.5^{\circ} \mathrm{C}$-WBGT intervals. For example, the TLVs for moderate work are: $28.0^{\circ} \mathrm{C}-\mathrm{WBGT}$ for $75-100 \%$ work pace, $29.0^{\circ} \mathrm{C}$-WBGT for $50-75 \%$ work pace, $30.0^{\circ} \mathrm{C}$-WBGT for $25-50 \%$ work pace, and $31.5^{\circ} \mathrm{C}$ WBGT for 0-25\% work pace (ACGIH, 2013, p. 208).

\subsection{Clothing effects}

Clothing influences the effect of heat stress on heat strain through thermal insulation $\left(I_{c l}\right)$ which affects the transfer of heat through radiation and conduction, and water vapour permeability $\left(I_{\mathrm{mst}}\right)$ 
which affects heat loss through evaporation. ACGIH provided WBGT adjustment values for several standard clothing ensembles, e.g. $1^{\circ} \mathrm{C}-\mathrm{WBGT}$ is to be added to the reading of the ambient environment for wearing polyolefin coveralls (ACGIH, 2009; Bernard et al., 2005; O'Connor \& Bernard, 1999). The adjustment values are determined by empirical testing through climatic chamber experiments. Whilst providing reference values for some typical hot working environments, this approach produces limited options without flexibility, as, for example, inclusion or removal of gloves or boots from the ensemble can make a significant difference to the adjustment value.

Other than the empirical approach, the rational approach estimates clothing effects on heat strain by taking explicit account of $I_{c l}$ and $I_{m s t}$ in the human thermal balance equation (e.g. Givoni \& Goldman, 1972, 1973; Haslam \& Parsons, 1988). The estimation is further improved by including the influence of air movement on clothing thermal insulation characteristics in the update of the ISO 7933 programme (ISO 7933, 2004; Parsons et al., 1999). ISO 9920 (2009) provides a catalogue of the values of thermal insulation and vapour permeability of clothing ensembles and materials. As $I_{\mathrm{cl}}$ and $I_{\text {mst }}$ are open to modification in the computer program provided in ISO 7933, it is possible to test the thermal performance of different types of PPE by varying the option parameters. The rational model is therefore a cost effective and more valid approach to estimation of the clothing effect on heat stress.

\section{Coping with boundary conditions: individual factors}

\subsection{Acclimatization}

Acclimatization is the physiological adaptation of the human body to heat. Acclimatised workers suffer less heat strain (ISO 7243, 1989; OSHA, 1999b) through improved ability in reserving sodium in sweat and a more efficient heat dissipation system, and are therefore more tolerant to heat stress(cf. ACGIH, 2009). Tian et al (2011) found that an acclimatized person exhibits higher sweat rate, and lower heart rate, oral temperature, systolic blood pressure, and levels of fatigue, than an unacclimatised person. ACGIH(2012) sets two sets of thresholds, one as TLV for an acclimatized person, another as AL (Action Limit) for an unacclimatised person, with a difference of around $3^{\circ} \mathrm{C}$ WBGT. ISO 7243 (1989) reference values have a $1-2^{\circ} \mathrm{C}-$ WBGT difference between acclimatized and unacclimatized subjects. Acclimatization can be achieved by a controlled gradually increasing exposure to heat over a period of 3-7 days (ISO 7243, 1989; NIOSH, 1986). In practice, a three-day acclimatisation protocol is usually recommended as an administrative measure for heat stress management (CSAO, 2007; OSHA, 1999b).

If people are not exposed to the same level of heat stress for one or two weeks, acclimatisation can be totally lost $(\mathrm{ACGIH}, 2009)$. Therefore whenever workers move from one area to another (as with migrant workers) one cannot assume they will immediately be fully acclimatised. An adaptation procedure still needs to be practised as appropriate, although environmental thresholds may vary from region to region, and need to be identified based on empirical data of the regional climate and the working population.

\subsection{Personal health factors}

All existing heat indices assume an average or standard person who is heat acclimatized, adequately hydrated, unmedicated, fit and healthy. Whilst thresholds for a standard person help identify safe boundaries for a heat stress management system, individual differences blur the boundaries and make it necessary to enable individual coping at boundaries. Over a century's physiological research has identified personal factors associated with human vulnerability to heat stress as: age, gender, race, physical fitness, obesity, hydration state, medication, personal acclimatization capacity, alcohol intake, smoking, etc. (Aaron, 1911; Andersen et al., 1976; Engell et al., 1987; Frye \& Kamon, 1981; Gun \& Budd, 1995; Havenith \& Middendorp, 1990; Inoue \& Shibasaki, 1996; Kawahata, 1960; Lind, 
et al., 1957). Individual smoking habits have a negative impact on heat dissipation ability by impairing body aerobic capacity, ineffective ventilation of the lungs, and induce cardiovascular diseases such as high blood pressure which expose the individual to heat induced illness (Benowitz et al., 1982; Grassi et al., 1992; Raven et al., 1974). Poor sleep quality is regarded as a factor contributing to the likelihood of heat related disorders yet no evidence has been found for this link. The assumed vulnerability is probably due to the link between restlessness and fatigue (Dawson \& Reid, 1997). The difficulty of quantifying the exact consequence of each factor and the interaction effect among the factors make it even more complicated to predict correlations between various factors. For example, whilst it is commonly believed that alcohol consumption and obesity aggravate heat strain, in some studies it was found that workers who are overweight and drank more alcohol exhibited less physiological strain in heat and were more productive (Gun \& Budd, 1995). Reverse results were attributed to individual workers' levels of skill at their work. Observation of skilled workers indicates they exhibit less stress and manage their work processes in a calm, controlled manner and so appear to expend less energy (reduced metabolic rate). More recent work such as Bethea and Parsons (2002) included personal job skill level as an item in their personal heat risk assessment checklist.

Medical and biological factors that contribute to the individual difference in heat tolerance are summarised in Appendices A and B. The Appendices are summarised from health screening items recommended by Ramphal-Naley (2012), American Academy of Family Physicians (AAFP, 2005), Federal Motor Carrier Safety Administration (FMCSA, 2010), Health and Safety Executive of the UK (Bethea \& Parsons, 2002), ISO 12894 (2001) and ACGIH(2009). The length of the lists excludes the possibility for a prescriptive benchmark or formula for calculation of the effect of personal health factors. In most existing guidelines, individual health factors are either ignored or addressed by a single notice: "NEVER ignore anyone's signs or symptoms of heat-related disorders" on top of all preventive measures. The risks, however, can be minimized by induction health screening, giving special attention to identified risky individuals, and empowering individual workers to cope through improving personal health awareness and training in other coping skills.

\subsection{Self-paced work on construction sites}

Hot weather triggers the body's autonomous adaptation mechanism such that workers slow down their work pace to prevent the body from excessive strain (Ebi et al., 2005; Kjellstrom et al., 2009b). In an actual work setting, Miller et al (2011) reported that "few workers will voluntarily work at a pace that requires sustaining an average heart rate $>110 \mathrm{bpm}$ for any length of time". Similarly, Parikh et al (1978) found that ceramic workers automatically adjust their pace of work under heat stress such that no cumulative risk is found in workers' heart rate record throughout a work shift. This mechanism is expected to lead to a natural reduction in labour productivity (Miller, et al., 2011). Hot weather is thus considered to be associated with low productivity and subsequent cost overruns (e.g. Frimpong, et al., 2003). The claim is further added by a finding from an experimental study that electrical journeymen in their routine tasks can only achieve full efficiency within a range of temperatures, i.e., $40-70^{\circ} \mathrm{F}$ and relative humidity below $80 \%$, while work performance significantly decreases when the temperature is above $80^{\circ} \mathrm{F}$ (NECA, 2004). The recognition of autonomous adaptation leads to the recommendation of self-regulation as an effective measure to manage heat stress (Miller, et al., 2011).

However, the claimed productivity reduction by self-pacing at work may indeed be fallacious in that an un-fatigued worker is less likely to make a mistake and have to redo work. Moreover, this view neglects to take into account management's responsibility to take environmental conditions into account in planning and costing work. Most importantly, self-paced work in construction sites is often subject to a number of constraints that have been reflected in the frequent occurrence of heat induced illness on construction sites during summer. 
The pressure for project progress is reported to pace work activities. Gertner et al (1984, p. 135) observed a contrast in heat strain between two groups of metal workers in a desert setting, one under progress pressure and the other without. Whilst the group free of progress pressure "tend to regulate their heart rate at the expense of effort and productivity...", the heart rate record of the other group in an urgent task "was high as would have been expected" (p.144). In some cultural regions, self-pace is constrained by workers' worry about losing wages or bonus, peer pressure or self-perception of effectiveness. Taking this into account, Mairiaux and Malchaire (1985) put conditions in their recommendations on self-regulation, "the task has no urgent character and does not involve productivity incentives, and that the workers are well trained to their job" (p.85). The effectiveness of self-regulation is also constrained by workers' awareness and knowledge of heat stress and even by the physiological factors of individual difference (e.g. sense of thirst). Therefore workers need to be explicitly empowered and trained to ensure the effectiveness of self-regulation.

The preceding discussion has four implications for construction projects. Firstly, the reduction of labour productivity in summer should be budgeted for in the project planning stage. However, much of the evidence for decreased productivity is hearsay; if proper engineering controls are introduced to reduce heat stress then productivity may not reduce at all. Secondly, a mandatory work-rest regimen should be implemented when particular climatic conditions arise. Thirdly, the constraints on allowing self-paced work should be explicitly removed when specific conditions are reached to ensure the effectiveness of self-regulation in heat stress risk management. Finally, heat risk management must be simplified and integrated into the overall safety management system of construction projects so that measures can be effectively implemented by laymen.

\section{Methodologies in the prediction of maximum allowable exposure duration $\left(D_{\text {lim }}\right)$ and recovery time $(R T)$}

Time plays an important role in the process of heat accumulation in the body and development of physiological disorders. The prediction of Maximum Allowable Exposure Duration $\left(D_{\text {lim }}\right)$ can thus give reference values for determining the continuous work time (CWT) in setting work-rest regimens. Three methodologies are identified in prediction of $D_{\text {lim; }}$ the empirical approach; the rational approach; and other approaches.

\subsection{The empirical approach}

The empirical approach has been adopted in the development of the TLVs (ACGIH, 2012) and the reference values of ISO 7243 (1989). The method is based on direct recording of a series of environmental conditions and subsequent heat strain measures taken from participants to establish the relationship between WBGT and metabolic rate, $D_{\lim }$ or clothing effects for categorised workload levels. Thresholds in WBGT are developed from direct observation of boundary conditions. Whilst this methodology does provide a basis for producing usable guidelines, the problem is that data from most empirical studies are taken from experiments in a climatic chamber which serves to simulate microclimates in a controlled manner with prescribed parameters (e.g. Ashley, et al., 2008; Bernard \& Ashley, 2009; Gonsalez et al., 2006; Zhao et al., 2009). An intrinsic constraint with climatic chamber simulation is that it cannot simulate variations in solar radiation. Besides, combinations of options of temperature, humidity, wind speed, and metabolic rate simulated in laboratory are far too limited compared with what happens in the natural environment and in real work (ACGIH, 2009; McNeill \& Parsons, 1999). Hence, thresholds produced from climatic chamber data cannot adequately address construction site conditions to guide a heat risk management system. More authentic data from the field which covers a full range of local weather conditions and working options are needed to underpin threshold-based industrial guidelines. 


\subsection{The rational approach}

The rational approach explores the relationship between $D_{\lim }$ and heat stress through taking explicit account of the internal mechanism of the human thermoregulation process (e.g. Brake, 2002; Givoni \& Goldman, 1973; ISO 7933, 1989; 2004 etc.). Heat storage rate in the body is estimated with the human thermal balance equation (ASHRAE, 2009; ISO 7933, 2004):

$$
\mathrm{M}-\mathrm{W}=\mathrm{C}_{\text {res }}+\mathrm{E}_{\text {res }}+\mathrm{K}+\mathrm{C}+\mathrm{R}+\mathrm{E}+\mathrm{S}
$$

where $M$ represents metabolic rate; $W$, effective mechanical power; $C_{\text {res, }}$, heat exchanges in the respiratory tract by convection; $\mathrm{E}_{\mathrm{re}}$, by evaporative; $\mathrm{K}$, heat exchange on the skin by conduction; $\mathrm{C}$, by convection; R, by radiation; E, by Evaporation, S, body heat storage.

The Predicted Heat Strain (PHS) model was jointly developed by eight major European laboratories (Malchaire, et al., 2001; Malchaire, 2006; Parsons, 1995) as a result of extensive review, testing, revision and reconstruction of the Required Sweat Rate model (ISO 7933, 1989). The updated PHS model estimates heat strain and $D_{\lim }$ from the input of the air temperature, vapor pressure (corresponding to humidity), mean radiant heat (linked with Globe Temperature), air velocity (wind speed), metabolic rate, clothing effect ( $I_{c l}$ and $I_{m s t}$ and their corrective factors), body size, posture, and wind direction. The required evaporative heat flow, skin wettedness, and the sweat rate for maintaining body thermal balance are calculated and compared with their predicted counterparts to estimate the rate of heat storage manifested in the increase of the skin and body core temperature (ISO 7933, 2004; Parsons, 1995).

ISO 7933 (2004) enables prediction of $D_{\lim }$ for any combination of environmental and metabolic heat stress and clothing effects. It also allows sensitivity analysis for testing the impact of specific parameters by standardizing others. Due to the complexity of the calculation process, it is implemented as a "black box" conducted by a computer program (Malchaire, 2006, p. 386), which is now available with ISO 7933 (2004). It is the best validated model thus far, backed by 672 laboratory experiments and 237 field experiments (Malchaire, 2006).

Other than the empirical and the rational approaches, it is interesting to note some recent research attempts to predict $D_{\lim }$ with all-in-one multiple regression models (e.g. Chan et al., 2011; 2012b; 2012c). Research output founded on this approach should be treated with caution. In fact it has long been recognized that the relationship between heat stress and heat strain is not linear (Malchaire, 1991). Furthermore, the combination of the large array of personal health and lifestyle factors and their complex interaction effects, as discussed in Section 3.6, is far beyond the predictive power of a multiple regression model. An important warning to heed with this approach is that "association does not imply causation", variables incorporated into the regression model should be based on theory and sound evidence. Methodological issues such as levels of study need to be consistent before they can be taken as a useful reference for future research.

Overall, examination of various approaches convinces us that the rational approach, more specifically the PHS model (ISO 7933, 2004), has distinct advantages in terms of accuracy and validity. Its simulation and prediction power provides a cost-effective way of predicting $\mathrm{D}_{\lim }$ in a much larger sample size than can be done with the empirical approach with data from climatic chamber experiments. So far a feature that has thwarted its application in a wider area is that it has to be applied through a complicated procedure that is incomprehensible to laymen and is not convenient for instant decision making. However, there is potential to simplify its application by incorporating the four environmental parameters in the WBGT index and developing localized thresholds with empirical data. To be able to extend these rational methods to large scale on site surveys provides the opportunity to identify and quantify variations in tolerance of workers in different regions and circumstances under solar radiation and so builds on the scientific nature of the prior research by taking it out into the field. The authors present a protocol for this methodology in a separate paper. 


\subsection{Time-Weighted-Average (TWA) and recovery time}

The recovery time needed after a period of continuous work is not separable from work pace as discussed in Section 3.3. For example, a 50\% work regimen specified in ACGIH (2013) guidelines can be operated as a regimen of resting for one hour after one hour's work, or, it can be operated as resting for five minutes after every five minutes' work. The former is often interpreted as a clear continuous work time and a needed recovery time, whereas the latter is more apt to be interpreted as a relaxation of work pace. The needed recovery time, assuming work and rest places have equal WBGT, is estimated by the effective metabolic rate which allows the person to practice unlimited continuous work without suffering excessive heat strain. The effective metabolic rate is calculated as a Time-Weighted-Average (TWA) among the metabolic rates in the work and rest portion of the examined period with the following equation (ACGIH, 2009; Parsons, 2006).

$$
M_{\text {eff }}=\left[\left(M_{\text {work }} \times t_{\text {work }}\right)+\left(M_{\text {rest }} \times t_{\text {rest }}\right)\right] /\left(t_{\text {work }}+t_{\text {rest }}\right)
$$

In a fluctuating thermal environment, the effective WBGT is to be calculated by Time-WeightedAverage (TWA) for each 60 or 120 minutes' work cycle with the following equation.

$$
W_{\text {Bff }}=\left[\left(W_{\text {efG }} T_{\text {work }} \times t_{\text {work }}\right)+\left(W B G T_{\text {rest }} \times t_{\text {rest }}\right)\right] /\left(t_{\text {work }}+t_{\text {rest }}\right)
$$

Based on the concept of TWA, the general principle of heat stress management is to bring down the average of either metabolic rate or WBGT (Brake \& Bates, 2002). The former can be lowered by either adjusting work pace or implementing a mandatory work-rest regimen; the latter can be controlled by implementing engineering measures at the workplace (such as air cooling, air movement generated by fans and shading in external environments) and lowering WBGT at the rest place.

Malchaire (1991) developed an exponential averaging method to improve the precision of prediction of heat strain in the rational model over the TWA method, which is now incorporated into the PHS model in ISO 7933 (2004). Whilst exponential averaging improved the validity of PHS model, the TWA remains a widely used and easy-to-interpret the method of monitoring heat stress over a particular length of time.

A recent empirical study (Chan et al., 2012a) attempted to predict recovery time from Physical Strain Index (PSI) (Moran et al., 1995), a heat strain index combining body core temperature and heart rate, with a multiple regression equation. Apart from the problems with empirical approaches in climatic chambers (Section 4.1 and 4.2), the predicted recovery time is based on one single option of environmental conditions, i.e., $30^{\circ} \mathrm{C}$ air temperature and $75 \%$ relative humidity. The variation of this empirical relation in different thermal environments has not been reported thus far but, as fatigue has been seen to be non-linear in laboratory experiments, variance with environmental parameters is also likely to be non-linear.

\section{Heat stress risk management protocols in context}

Two globally influential heat risk management protocols with similar rationales and procedures are recommended by ACGIH(ACGIH, 2009, 2012, 2013) and International Standard Organisation (ISO) (ISO 7243, 1989; Parsons, 1995, 2003, 2006). The recommended heat risk management guidelines start from an initial subjective judgment of the existence of heat stress, followed by monitoring of the workplace WBGT, adjustment according to the clothing effect, comparison to the TLVs (ACGIH, 2012) or reference values (ISO 7243, 1989). Conditions of workplace WBGT exceeding the thresholds are then subject to detailed analysis with ISO 7933 (2004) or other rational models for prediction of heat strain. Meanwhile general control measures including training, promotion of healthy lifestyle, acclimatization, hydration, and health screening are to be implemented prior to the use of the WBGT thresholds. When excessive heat strain is predicted, direct monitoring of heat strain is to be implemented (ISO 9886, 2004), the result of excessive heat strain triggers job specific control 
measures, including engineering measures (which, in the case of construction sites, includes shelters at work or rest places to reduce radiant heat gain, ventilation in indoor working environments, mechanical aids to reduce metabolic heat gain, air-conditioned rest rooms, provision of drinking water or sports drinks, etc.), work-rest regimens, rescheduling of work or other administrative measures, to mitigate the heat stress risk.

Such a protocol isolates risk management from the production process. The consequence of such modeling approach is that rules be violated or ignored by operational staff under production constraints such as workload or time pressure (Rasmussen, 1997), Specifically on construction sites, a number of issues are apparent. Firstly, the protocol assumes the risk to be analysed by a specialist and decisions made by professional judgment. In the actual operation system of construction projects such decisions will have to be made by local players, the lay site supervisory staff. In that case the complicated procedures to be followed before reaching a managerial decision are neither effective nor efficient. A quick change of weather conditions and the need for instant decision making in order to mitigate risks on construction sites is in sharp contrast to the conditions in which the protocol is intended to be used. For this reason, although the ACGIH and the ISO thresholds systems are recommended for initial screening only, most existing guidelines simply take them as action triggering benchmarks (e.g.CSAO, 2009; CSAO, 2010). Not surprisingly they were found to be over-conservative and involve a high compliance cost, such that the thresholds are regarded by many employers as being a "security biased over-evaluation" (Budd, 2008).

In the real world a climatic heat risk management plan is the practical negotiation of objectives derived from the understanding of occupational culture, social conflict, power balance, control and access to resources, compliance cost and the demands of productivity. From an organizational point of view, risk management is the rational and systematic handling of risks through identification, analysis, mitigation, control, and finally, financing of the inevitable losses (Chapman \& Ward, 2003; $\mathrm{PMI}, 2008$ ). The principle of risk management is to transfer the risk to the party who can best bear that risk. Thus when players at different stages of a construction project lifecycle perform their role in heat risk control, the timing of implementation of ACGIH specified risk mitigation measures will be totally different.

Construction work procedures and their available alternatives are part of the familiar context of frontline staff, who, in daily in-situ decision making do not use analytical reasoning but replace it with "a simple skill- and rule-based choice among familiar action alternatives" (Rasmussen, 1997, p. 187). Therefore a series of simplified action-triggering decision making tools will work and be adopted into the overall construction safety management plan. The tools must be usable and have to be based on regional research in order to be relevant, appropriate and effective whilst maintaining legitimacy and not infringing on local cultural norms and values (Parsons, 2013).

\section{Discussion}

\subsection{Research agenda for a climatic heat risk management study in a specific region}

The procedures and tools reviewed in this paper form the basis to analyse the typical summer working environment in a specific region and produce a decision making tool for frontline staff to mitigate heat stress risk on site. An action triggering threshold system can be calculated from empirical data collected on site during the summer months to trigger different levels of management action. WBGT has the convenience and validity to be a usable index for monitoring the thermal environment of construction sites. The development of such a system should address the regional climate, characteristics of the work and the workforce, evaluation of the clothing effect, and define scientifically assessed work-rest regimens to improve safety, health and efficiency. Basically three levels of environmental thresholds are needed: 
- The point for implementing engineering controls to maintain the normal work-rest regimen;

- The point for implementing mandatory breaks; and

- The point for enabling self-paced work

The decision on setting three levels of work-rest regimens should be based on a compromise between the CWT and the environmental thresholds informed by data from site studies. Whereas

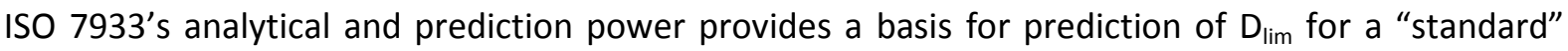
person working in representative weather conditions of a region, the three-level thresholds system must be further validated with existing practices on site in a representative sample of each regional occupational population. Following the set-up of the environmental thresholds, boundaries between roles of actors in the whole system will be go through a process of change which should be addressed during system development (Leveson, 2011). This can be an area of value for further research effort.

Rasmussen (1997) identifies six key factors for developing a risk management system on a specific hazard source, including: (1) identification of the overall control structure, (2) relevant actors and (3) their objectives and accountability, (4) available information on production objectives and safety boundaries for each role, (5) actors' competence and (6) commitment. Whereas objectives refer to values, process optimization criteria and constraints; competence includes both explicit and tacit knowledge specific to a local context; commitment concerns priorities and readiness to take the required control action. Leveson (2011) differentiates the concepts of hierarchical safety control structures for system development and for system operations and establishes a feedback loop between the two structures. Within the scope of a construction project organsiation, an initial attempt at identifying actors and their roles in heat risk management along a construction project lifecycle is presented in Table 1. Within the existing control structure, the critical factor for success of the risk management plan is to match the accountability of each actor in the system with the right information, competence and commitment.

Table 1 Actors and their roles in a construction project organisation along a project life cycle

\begin{tabular}{|c|c|c|}
\hline Stages & Actors & Objectives and accountability \\
\hline \multirow{2}{*}{$\begin{array}{l}\text { Tendering \& } \\
\text { contracting }\end{array}$} & \multirow{2}{*}{ Client } & Set achievable targets in terms of timing \& budget \\
\hline & & Mandate heat stress management in contractual provisions \\
\hline \multirow{2}{*}{ Design } & \multirow{2}{*}{ Consultants* } & Inform clients of heat stress issues which may arise during construction stage \\
\hline & & Documentation of heat stress black spots \\
\hline \multirow{4}{*}{$\begin{array}{l}\text { Project } \\
\text { planning }\end{array}$} & \multirow{4}{*}{$\begin{array}{l}\text { Contractor: } \\
\text { Project } \\
\text { manager }\end{array}$} & Schedule work to cooler period/place or arrange rotation \\
\hline & & Substitute heat generating plant; provide mechanical aids \\
\hline & & Establish surveillance and risk assessment system \\
\hline & & Provide hygiene facilities, staff, health screening and first-aid, training, etc. \\
\hline \multirow{8}{*}{ Construction } & $\begin{array}{l}\text { Planner/saf } \\
\text { ety manager }\end{array}$ & $\begin{array}{l}\text { Day-to-day planning of work; assessment of goals; dynamic heat risk } \\
\text { assessment }\end{array}$ \\
\hline & \multirow{2}{*}{\begin{tabular}{|l|} 
Site \\
supervisor \\
\end{tabular}} & Decision making on mandatory breaks \\
\hline & & Enable self-pacing \\
\hline & \multirow{3}{*}{ Worker } & Follow mandatory breaks; drink water; take rest; etc \\
\hline & & Self-pace upon empowerment; self-treatment on early signs of heat illness \\
\hline & & Keep an eye on coworkers' symptoms \\
\hline & $\begin{array}{l}\text { Safety } \\
\text { officer }\end{array}$ & $\begin{array}{l}\text { Enforcement, documentation, feeding back performance of operation of the } \\
\text { CHRM system }\end{array}$ \\
\hline & $\begin{array}{l}\text { Insurance, } \\
\text { Contractor } \\
\text { \& Client }\end{array}$ & Financing inevitable loss, compensation \\
\hline
\end{tabular}

\footnotetext{
* Consultants include Architect
} 


\subsection{Estimation of productivity reduction in heat}

The implementation of a mandatory work-rest regimen is likely to be perceived to be reducing productivity and thus resisted by the employers, as well as workers if the cost of productivity reduction leads to a drop in take home pay, as it might in Asia for example. However, as argued above, the slowing down of work pace and reduction of productivity may or may not occur due to actions taken to reduce environmental heat stress. Stakeholders need to evaluate field data to judge whether a heat stress initiative actually leads to a reduction in productivity (based on proposed work-rest regimens on site). Current research, to be reported later, is addressing this issue

Existing methods of estimating productivity reduction due to heat stress focus on the allowable metabolic rate in proportion to the full metabolic rate (Brake, 2002). This estimation is based on an assumption of a standard person and expected adaptation; in reality this may vary across regions and cultures and the estimation may be over conservative in that it underestimates both the potential of the human body to acclimatise and the constraints of self-pace in the social and managerial environment of the workplace. The gap between actual productivity reduction and the theoretical reduction are thus being empirically researched. The consequences of additional breaks on productivity, whether they will reduce productivity through lost working time or improve productivity through improved efficiency, are currently being monitored and analysed.

\subsection{Possible changes in accident patterns and mitigation of risk}

The implementation of the mandatory work-rest regimen will see a change in accident patterns but not necessarily an immediate drop in accident rates without follow-up monitoring and prevention measures. Accidents caused by physical and mental fatigue and heat-induced illness should be reduced. As a note of warning, which requires management attention before implementation, breaks can cause a loss of concentration, consequently, accidents increase before the break when workers rush to get the work done and after breaks when workers are still re-concentrating themselves (Ling et al., 2009; Rowlinson, 1997). Further development in the system should look into strategies that enable effective coping at boundary conditions.

\subsection{Implications for accident prevention}

Preceding discussions imply that the performance of work activities befitting safety and accident prevention are a continuing and dynamic process. The key to achieving these objectives lies with workers' concentration. The cognitive condition of concentration can be viewed through the concept of mindfulness (Weick et al., 1999). Mindful work organisation and performance can be achieved through the improvement of workers' alertness to and awareness of the details of the operations. An improved alertness on the part of the worker enables the detection of subtle changes of the contexts of the operating environments. It is clear that the implementation of mandatory breaks and self-paced work contribute towards engendering mindfulness not only of individual workers but also the group of workers who are constantly interacting on site and management.

Based on a taxonomy of dominant hazard sources, Rasmussen (1997) classified risk management into three general strategies: the empirical strategy being used in occupational safety such as in the construction industry; the evolutionary strategy being used in protection against particular accident process such as train collisions and aircraft accidents; the analytical strategy being used in high reliability organisations (HRO) (Weick, et al., 1999) such as nuclear power plants. Existing risk management in the construction industry commits its safety level to be "typically controlled empirically from epidemiological studies of past accidents" (p.198). Through adoption of a systemic plan driven by the threshold-based climatic heat stress guidelines, the way of operating a construction project organisation will closely resemble the concept of operating a HRO. HRO is characterized by a set of work processes that allow the operators to continuously operate under 
challenging conditions, maintain resilience, and help with the recovery process (cf. Weick \& Sutcliffe, 2007). Learning (cf. Senge, 2006), self-organising (Rasmussen, 1997), and continuous adaptation are the central tenet of HRO. The key to improvement of safety in HROs is to decouple reliability from safety, the former indicates to what extent an individual player in the system follows the specified procedure whereas the latter is a property of the system as a whole (Leveson, 1995, 2011). Safety performance is thus to be achieved through integration of the otherwise conflicting goals of production and safety, or of the sometimes conflicting safety goals in tackling different hazard sources, through careful devises of safety constraints at different levels of the hierarchal control structure of the system (Leveson, 2011). Effective heat stress management takes a thorough review of the operating system of a construction project organisation and its potentially conflicting goals before coordinating them within a project hierarchical safety control structure which embeds the heat stress management system. The structure is then to be devised to facilitate timely human (i.e. the workers) adjustment to their work environment through the process of mindfulness. Mindfulness enables workers' continuous interactions in a work unit as they develop shared cognition of the situation that they encounter and their capabilities to act. This collective capability can potentially prevent accidents (cf. Sutcliffe, 2011). This area is ripe for further research to enable advancement in the field of construction accident prevention.

Decades ago twelve research needs on heat stress were identified by the NIOSH (1986), most of which have been extensively explored thus far, except for the topic of "accidents and heat stress". Little empirical research is evident in this stream, which may, in part, be due to lack of documentation of weather conditions in accident investigation in certain jurisdictions, although research does show a peak in accident occurrence in hot and humid weather in Hong Kong, for example. The development of regional heat stress thresholds and establishment of climatic heat risk management systems provides an infrastructure for documentation and data collection for systematic analysis of the relationship between heat stress and accident occurrence and causation.

\section{Conclusion}

This paper has reviewed past and current practice in formulating heat stress guidelines and trigger points for action. The aim of this review is to inform research aimed at developing appropriate measures for specific regions and the construction industry generally. The uniqueness of the industry is its location - outdoors under the elements, particularly solar radiation in the summer. Such conditions are not easily simulated in a chamber. In conducting this review a whole range of methodologies have been reviewed and a set of guidelines to direct further research have been developed.

The ongoing research has the objective of determining appropriate trigger points for the implementation of a heat stress management system and within that system a work-rest regime determined based on empirical measurements in the field. Importantly, and to ensure "buy-in" by industry, it is recognised that there may be some apparent reduction in productivity during interventions aimed at reducing heat stress in workers. However, initial site studies in Hong Kong have indicated that this productivity reduction may well be insignificant due to the improvements in working environment brought about by engineering controls, heat stress triggers and proposed rest measures. This aspect of heat stress management is vitally important in gaining industry support for new heat stress guidelines and so will be an important aspect of the research plan.

What is evident from this review is that there has been a lack of research into real life industrial situations and so an inability to formulate effective guidelines and trigger points for specific regional locations and industries. This is not a criticism of previous work because this work has actually laid the foundations for a rational, focused and justifiable approach to determining guidelines and thresholds outside of the chamber and in real, industrial settings around the world. Thus, what the authors have done is to take cognisance of past research and examine them in the light of systems 
thinking in formulating a way forward in order to determine thresholds and guidelines for workers in the construction industry, in our case based in Hong Kong and China. Hence, future publications in this area will report on justifiable, carefully constructed live studies, in this particular region and will report on the formulation of very specific approaches to heat stress management for this region and their effects on productivity.

\section{Acknowledgement}

The research is partially supported by the Safety Sub-Committee of Hong Kong Construction Industry Council, Seed Funding Grant for Applied Research (Project No. 201109160027) and Leung Kau Kui Research and Teaching Endowment Fund (Project No. 201011165012) at the University of Hong Kong, and the Ministry of Education Key Laboratory of Eco-environments in Three Gorges Reservoir Region at Chongqing University.

[Note]

${ }^{1}$ The empirical figures are based on a field study by the authors with environmental and physiological data collected from 26 construction sites in Hong Kong over 69 days during the summer of 2011. Sample sizes are 207 for workers' sample; 92 for managers and supervisors' sample. Details will be reported in a separate paper.

\section{References}

AAFP. (2005). Information for your family doctor - heat stress and heat stroke: what you should know. American Family Physician, 71 (11), 2141-2142.

Aaron, H. (1911). Investigation into the action of the tropical sun on men and animals. Philippine Journal of Science, 6, 101.

Abdelsalam, H. M. E., \& Gad, M. M. (2009). Cost of quality in Dubai: an analytical case study of residential construction projects. International Journal of Project Management, 27, 501-511.

Abu Dhabi EHS Center. (2012). Technical Guideline: Safety in the Heat (Abu Dhabi EHSMS Regulatory Framework). UAE: Abu Dhabi Environment, Health and Safety Center.

ACGIH. (2009). Heat Stress and Heat Strain: TLV® Physical Agents 7th Edition Documentation Cincinnati: American Conference of Governmental Industrial Hygienists.

ACGIH. (2012). 2012 TLVs ${ }^{\circledR}$ and BEIs ${ }^{\circledR}$ - Based on the Documentation of the Threshold Limit Values for Chemical Substances and Physical Agents \& Biological Exposure Indices. American Conference of Governmental Industrial Hygienists: Publication \#0110.

ACGIH. (2013). 2013 TLVs ${ }^{\circledR}$ and BEIs ${ }^{\circledR}$ - Based on the Documentation of the Threshold Limit Values for Chemical Substances and Physical Agents \& Biological Exposure Indices. Cincinnati: American Conference of Governmental Industrial Hygienists.

AIOH. (2003). Heat Stress Standard \& Documentation Developed for Use in the Australian Environment (Developed by Ross Di Corleto, Gerry Coles and Ian Firth). Tullamarine: The Australian Institute of Occupational Hygienists Inc.

Andersen, I., Jensen, P. L., Junker, P., Thomsen, A., \& Wyon, D. P. (1976). The effects of moderate heat stress on patients with ischemic heart disease. Scandinavian Journal of Work, Environment \& Health, 2 (4), 256-268.

Ashley, C. D., Luecke, C. L., Schwartz, S. S., Islam, M. Z., \& Bernard, T. E. (2008). Heat strain at the critical WBGT and the effects of gender, clothing and metabolic rate. International Journal of Industrial Ergonomics, 38, 640-644.

ASHRAE. (2009). ASHRAE Handbook - Fundamentals. Atlanta, GA: American Society of Heating, Refrigerating and Air-Conditioning Engineers, Inc.

Bedford, T. (1946). Environmental warmth and its measurement. Medical Research Council War Memo No. 17. London: H. M. S. O.

Belding, H. S., \& Hatch, T. F. (1955). Index for evaluating heat stress in terms of resulting physiological strains. Heating, Piping, Air Conditioning, 27, 129-135.

Bell, S. (2012). Commissioner for Mine Safety and Health: Queensland Mines Inspectorate Annual Performance Report 2011-12. Queensland, Australia: Queensland Mines Inspectorate, Department of Natural Resources and Mines, State of Queensland.

Benowitz, N. L., Jacob III, P., Jones, R. T., \& Rosenberg, J. (1982). Interindividual variability in the metabolism and cardiovascular effects of Nicotine in man. The Journal of Phyamacology and Experimental Therapeutics, 221 (2), 368-372. 
Bernard, T. E. (1999). Heat stress and protective clothing: an emerging approach from the United States. Annals of Occupational Hygiene, 43, 321-327.

Bernard, T. E., \& Ashley, C. (2009). Short-term heat stress exposure limits based on wet bulb globe temperature adjusted for clothing and metabolic rate. Journal of Occupational and Environmental Hygiene, 6 (10), 632-638.

Bernard, T. E., Luecke, C. L., Schwarts, S. W., Kirkland, K. S., \& Ashley, C. D. (2005). WBGT clothing adjustment for four clothing ensembles under three relative humidity levels. Journal of Occupational and Environmental Hygiene, 2, 251-256.

Bethea, D., \& Parsons, K. C. (2002). The development of a practical heat stress assessment methodology for use in UK industry (HSE Research Report 008). London, UK: Health \& Safety Executive.

Blagden, C. (1775a). Experiments and observations in a heated room. Philosophical Transactions of the Royal Society of London, 65, 111-123.

Blagden, C. (1775b). Further experiments and observations in a heated room. Philosophical Transactions of the Royal Society of London, 65, 484-494.

Brake, D. J. (2002). The Deep Body Core Temperatures, Physical Fatigue and Fluid status of Thermally Stressed Workers and the Development of Thermal Work Limit as an Index of Heat Stress. PhD thesis, School of Public Health, Curtin University of Technology, Australia.

Brake, D. J., \& Bates, G. P. (2002). A valid method for comparing rational and empirical heat stress indices. The Annals of Occupational Hygiene, 46 (2), 165-174.

BS EN 27243. (1994). Hot Environments - Estimation of the Heat Stress on Working Man, Based on the WBGTIndex (Wet Bulb Globe Temperature) (ISO 7243: 1989). BSI: The British Standard Institution.

Budd, G. M. (2008). Wet-bulb globe temperature (WBGT) - its history and its limitations. Journal of Science and Medicine in Sport, 11, 20-32.

Chan, A. P. C. (2012). From Heat Tolerance Time to Optimal Recovery Time - A Heat Stress Model for Construction Workers in Hong Kong. Presentation at Hong Kong Polytechnic University at 31 May 2012.

Chan, A. P. C., Wong, F. K. W., Wong, D. P., Lam, E. W. M., \& Wen, Y. (2012a). Determining an optimal recovery time after exercising to exhaustion in a controlled climatic environment: application to construction workers. Building and Environment, 56, 28-37.

Chan, A. P. C., Wong, F. K. W., Yam, M. C. H., Chan, D. M. W., Mok, E. C. M., Shea, G. Y. K., ... Dingsdag, D. A. (2011). A research framework for assessing the effects of heat stress on construction workers. Proceedings of 6th International Structural Engineering and Construction Conference, ISEC06 Modern Methods and Advances in Structural Engineering and Construction, Zurich, 21-26 June, 485489.

Chan, A. P. C., Yam, M. C. H., Chung, J. W. Y., \& Wen, Y. (2012b). Developing a heat stress model for construction workers. Journal of Facilities Management, 10 (1), 59-74.

Chan, A. P. C., Yi, W., Chan, D. W. M., \& Wong, D. P. (2012c). Using the Thermal Work Limit (TWL) as an environmental determinant of heat stress for construction workers. Journal of Management in Engineering (C) ASCE, doi:10.1061/(ASCE)ME.1943-5479.0000162.

Chan, M. (2011). Fatigue: the most critical accident risk in oil and gas construction. Construction Management and Economics, 29, 341-353.

Chapman, C., \& Ward, S. (2003). Project Risk Management: Processes, Techniques and Insights (2nd Edition). Chichester, West Sussex, England: Wiley.

Chia, C.-F., Changa, T.-C., \& Ting, H.-I. (2005). Accident patterns and prevention measures for fatal occupational falls in the construction industry. Applied Ergonomics, 36, 391-400.

Choudhry, R. M., \& Fang, D. (2008). Why operatives engage in unsafe work behavior: investigating factors on construction sites. Safety Science, 46, 566-584.

Corleto, R. D. (2011). Heat Exposure in Mining: Three Step Assessment Protocol. Presentation at the Industrial Seminar Risk Management of Heat Exposure, Organized by DEEDI Mines and the Health Improvement and Awareness Committee (HIAC), Department of Natural Resources and Mines, Queensland Government, 24 November 2011, South Brisbane, Available at http://mines.industry.qld.gov.au/safety-and-health/651.htm accessed at 11 November 2012.

CSAO. (2000). Heat Stress: Guidelines for Recognition, Assessment, and Control in Construction. Etobicoke: Construction Safety Association of Ontario.

CSAO. (2007). Construction Multi-Trades Health and Safety Manual. Etobicoke: Construction Safety Association of Ontario.

CSAO. (2009). Construction Multi-Trades Health and Safety Manual. Etobicoke: Construction Safety Association of Ontario.

CSAO. (2010). Construction Health and Safety Manual. Etobicoke: Construction Safety Association of Ontario. 
Dawson, D., \& Reid, K. (1997). Fatigue, alcohol and performance impairment. Nature, 388 (17 July 1997), 235237.

Ebi, K. L., Smith, J. B., \& Burton, I. (Eds.). (2005). Integration of public health with adaptation to climate change. New York: Taylor \& Francis.

Edwards, P. J., \& Bowen, P. A. (1998). Risk and risk management in construction: a review and future directions for research. Engineering Construction and Architectural Management, 5, 339-349.

Engell, D. B., Maller, O., Sawka, M. N., Francesconi, R. N., Drolet, L., \& Young, A. J. (1987). Thirst and fluid intake following graded hypohydration levels in humans. Physiology \& Behavior, 40 (2), 229-236.

EPA. (1993). A Guide to Heat Stress in Agriculture. United States: Environmental Protection Agency, EPA750-b-92-001.

FMCSA. (2010). Rules and Regulations from the Federal Motor Carrier Safety Administration. Available at http://www.fmcsa.dot.gov/rules-regulations/rules-regulations.htm.

Frimpong, Y., Oluwoye, J., \& Crawford, L. (2003). Causes of delay and cost overruns in construction of groundwater projects in a developing countries: Ghana as a case study. International Journal of Project Management, 21, 321-326.

Frye, A. J., \& Kamon, E. (1981). Responses to dry heat of men and women with similar aerobic capacities. Journal of Applied Physiology, 50, 65-70.

Gagge, A. P., Stolwijk, J. A. J., \& Nishi, Y. (1970). An effective temperature scale based on a simple model of human physiological regulatory response. ASHRAE Journal, to be added.

Garrett, J. W., \& Teizer, J. (2009). Human factors analysis classification system relating to human error awareness taxonomy in construction safety. Journal of Construction Engineering and Management, 135 (8), 754-763.

GB/T 4200. (2008). Classified Standard of Working in the Hot Environment (in Chinese). Bejing: General Administration of Quality Supervision, Inspection, and Quarantine and Standardization Administration, P. R. China.

GBZ 2.2. (2007). Occupational exposure limits for hazardous agents in the workplace Part 2: Physical agents (in Chinese) Beijing: Ministry of Health.

GBZ/T 189.7. (2007). Measurement of Physical Agents in Workplace Part 7: Heat Stress (in Chinese). Beijing: Ministry of Health, P. R. China.

GBZ/T 229.3. (2010). Classification of Occupational Hazards at Workplaces Part 3: Occupational Exposure to Heat Stress (in Chinese). Beijing: Ministry of Health, P. R. China.

Gertner, A., Isreli, R., \& Cassuto, Y. (1984). Effects of work and motivation on the heart rates of chronic heatexposed workers during their regular work shifts. Ergonomics, 27 (2), 135-146.

Gillen, M., \& Gittleman, J. L. (2010). Path forward: emerging issues and challenges. Journal of Safety Research, 41, 301-306.

Givoni, B., \& Goldman, R. F. (1972). Predicting rectal temperature response to work, environment, and clothing. Journal of Applied Physiology, 32 (6), 812-822.

Givoni, B., \& Goldman, R. F. (1973). Predicting heart rate response to work, environment, and clothing. Journal of Applied Physiology, 34 (2), 201-204.

Gonsalez, N. W., Bernard, T. E., Carroll, N. L., Bryner, M. A., \& Zeigler, J. P. (2006). Maximum sustainable work rate for five protective clothing ensembles with respect to moisture vapor transmission rate and air permeability. Journal of Occupational and Environmental Hygiene, 3, 80-86.

Grassi, G., Seravalle, G., Calhoun, D. A., Bolla, G., \& Mancia, G. (1992). Cigarette smoking and the adrenergic nervous system. Clinical and Experimental Hypertension, a14 (1-2), 251-260.

Gun, R. T., \& Budd, G. M. (1995). Effects of thermal, personal and behavioural factors on the physiological strain, thermal comfort and productivity of Australian shearers in hot weather. Ergonomics, 38 (7), 1368-1384.

Haslam, R. A., \& Parsons, K. C. (1988). Quantifying the effects of clothing for models of human response to the thermal environment. Ergonomics, 31 (12), 1787-1806.

Hatch, T. F. (1973). Design requirements and limitations of a single-reading heat stress meter. American Industrial Hygiene Association Journal, 64 (6), 66-72.

Havenith, G., \& Middendorp, H. v. (1990). The relative influence of physical fitness, acclimatization state, anthropometric measures and gender on individual reactions to heat stress. European Journal of Applied Physiology and Occupational Physiology, 61 (5-6), 419-427.

Helander, M. (1980). Safety challenge in the construction industry. Journal of Occupational Accidents, 2, $257-$ 263.

Houghten, F. C., \& Yagloglou, C. P. (1923). Determination of comfort zone with further verification of Effective Temperature within this zone. Journal of the American Society of Heating and Ventilating Engineers, 29, 515-536. 
HSE. (2012a). Heat Stress - Measuring - Heart Rate: available at http://www.hse.gov.uk/temperature/heatstress/measuring/heart.htm (accessed at 10 December 2012).

HSE. (2012b). Heat Stress - Wet Bulb Globe Temperature Index. Health and Safety Executive, UK: Available at http://www.hse.gov.uk/temperature/heatstress/measuring/wetbulb.htm (accessed at 13 December 2012).

Inoue, Y., \& Shibasaki, M. (1996). Regional differences in age-related decrements of the cutaneous vascular and sweating responses to passive heating. European Journal of Applied Physiology, 74 (1-2), 78-84.

IOSH. (1997). Manual of Labor Heat Illness Prevention (勞工熱危害預防手冊). Taiwan: Institute of Occupational Safety and Health (Available at: http://www.iosh.gov.tw/Book/other_public_Publish.aspx?P=50).

IPCC. (2007). Climate Change 2007: Impacts, Adaptation and Vulnerability. Contribution of Working Group II to the Fourth Assessment Report of the Intergovernmental Panel on Climate Change. Cambridge: Cambridge University Press.

ISO 7243. (1989). Hot Environments - Estimation of the heat stress on working man, based on the WBGT-index (wet bulb globe temperature). Geneva: International Standard Organisation.

ISO 7933. (1989). Hot Environments - Analytical Determination and Interpretation of Heat Stress Using Calculation of the Required Sweat Rate. Geneva: International Standard Organisation.

ISO 7933. (2004). Ergonomics of the thermal environment Analytical determination and interpretation of heat stress using calculation of the predicted heat strain. Geneva: International Standard Organisation.

ISO 9886. (2004). Ergonomics. Evaluation of thermal strain by physiological measurements. Geneva: International Standard Organisation.

ISO 9920. (2009). Ergonomics of the thermal environment - Estimation of thermal insulation and water vapour resistance of a clothing ensemble. Geneva: International Standard Organisation.

ISO 12894. (2001). Ergonomics of the thermal environment - Medical supervision of individuals exposed to extreme hot or cold environments. Geneva: International Standard Organisation.

Kawahata, A. (1960). Sex differences in sweating. In H. Yoshimura, K. POgata \& S. Itoh (Eds.), Essential Problems in Climatic Physiology. Kyoto: Nankodo.

Kjellstrom, T., Gabrysch, S., Lemke, B., \& Dear, K. (2009a). The 'Hothaps' programme for assessing climate change impacts on occupational health and productivity: an invitation to carry out field studies. Global Health Action (DOI: 10.3402/gha.v2i0.2082).

Kjellstrom, T., Ingvar, H., \& Lemke, B. (2009b). Workplace heat stress, health and productivity - an increasing challenge for low and middle-income countries during climate change. Global Health Action, 2, 47-52.

Leveson, N. G. (1995). Software: System Safety and Computers. Reading, MA: Addison-Wesley.

Leveson, N. G. (2011). Applying systems thinking to analyze and learn from events. Safety Science, 49 (1), 55 64.

Lin, R.-T., \& Chan, C.-C. (2009). Effects of heat on workers' health and productivity in Taiwan. Global Health Action (DOI: 10.3402/gha.v2i0.2024).

Lind, A. R., Hellon, R. F., Weiner, J. S., Jones, R. M., \& Fraser, D. C. (1957). Reactions of mines-rescue personnel to work in hot environments, National Coal Board, Medical Research Memorandum No. 1. London: N. C. B.

Ling, F. Y. Y., Liu, M., \& Woo, Y. C. (2009). Construction fatalities in Singapore. International Journal of Project Management, 27, 717-726.

Lu, S., \& Zhu, N. (2007). Experimental research on physiological index at the heat tolerance limits in China. Building and Environment, 42 (2007), 4016-4021.

Lundgren, K., Kuklane, K., Gao, C., \& Holmér, I. (2013). Effects of heat stress on working populations when facing climate change. Industrial Health, 51, 3-15.

Mairiaux, P., \& Malchaire, J. (1985). Workers self-pacing in hot conditions: a case study. Applied Ergonomics, 16 (2), 85-90.

Malchaire, J., Piette, A., Kampmann, B., Mehnert, P., Gebhardt, H., Havenith, G., . . Griefahn, B. (2001). Development and validation of the Predicted Heat Strain model. Annals of Occupational Hygiene, 45 (2), 123-135.

Malchaire, J. B. (1991). Predicted sweat rate in fluctuating thermal conditions. European Journal of Applied Physiology and Occupational Physiology, 63 (3-4), 282-287.

Malchaire, J. B. M. (2006). Occupational heat stress assessment by the Predicted Heat Strain model. Industrial Health, 44, 380-387.

Masterton, J. M., \& Richardson, F. A. (1979). Humidex: A Method of Quantifying Human Discomfort Due to Excessive Heat and Humidity. Downsview, Ontario: Environment Canada (UDC: 551.971.612.59).

McNeill, M. B., \& Parsons, K. C. (1999). The appropriateness of international heat stress standards for use in tropical agricultural environments. Ergonomics, 42 (6), 779 - 797.

Miller, V. S., \& Bates, G. P. (2007). The Thermal Work Limit is a simple reliable geat index for the proection of workers in thermallly stressful environments. Annal of Occupational Hygiene, 51 (6), 553-561. 
Miller, V. S., Bates, G. P., Schneider, J. D., \& Thomsen, J. (2011). Self-pacing as a protective mechanism against the effects of heat stress. The Annals of Occupational Hygine, 55 (4), 1-8.

Moran, D., Epstein, Y., Laor, A., Vitalis, A., \& Shapiro, Y. (1995). Predicting heart rate response to various metabolic rates, environments, and clothing. Journal of Applied Physiology, 78 (1), 318-322.

NASA. (2006). Ingestible Thermometer Pill Aids Athletes in Beating the Heat. available at http://spinoff.nasa.gov/Spinoff2006/hm_1.html, accessed at 31 Dec 2012.

NECA. (1974). The Effect of Temperature on Productivity. Washingtong D C: National Electrical Contractors Association.

NECA. (2004). The Effect of Temperature on Productivity. Washingtong D C: National Electrical Contractors Association.

NIOSH. (1972). Criteria for a Recommended Standard: Occupational Exposure to Hot Environments. Ohio: DHHS (NIOSH) Publication Number 72-10269.

NIOSH. (1986). Occupational Exposure to Hot Environments. Washington DC, USA: National Institute for Occupational Safety and Health, DHHS (NIOSH) Publication No. 86-113.

O'Connor, D. J., \& Bernard, T. E. (1999). Continuing the search for WBGT clothing adjustment factors. Applied Occupational and Environmental Hygiene, 14, 119-125.

Ontario Ministry of Labour. (2013). Heat Stress. Ontario: Occupational Health and Safety Branch, Ontario Ministry of Labour, Canada.

OSHA. (1999a). OSHA Technical Manual. Occupational Safety and Health Administration, United States Department of Labor: http://www.osha.gov/dts/osta/otm/otm_iii/otm_iii_4.html\#iii:4_1 accessed at 13 August 2011.

OSHA. (1999b). OSHA Technical Manual. Chapter 4, Heat Stress In OSHA Technical Manual. Washington, DC: Occupational Safety \& Health Administration, Accessed at http://www.osha.gov/dts/osta/otm/otm_iii/otm_iii_4.html.

OSHS. (1997). Guidelines for the Management of Work in Extremes of Temperature. Wellington, New Zealand: Occupational Safety and Health Service, Department of Labour, New Zealand.

Parikh, D. J., Ghodasara, N. B., \& Ramanathan, N. L. (1978). A special thermal stress problem in ceramic industry. European Journal of Applied Physiology, 40, 63-72.

Parsons, K. (2013). Occupational health impact of climate change: current and future ISO standards for the assessment of heat stress. Industrial Health, 51, 86-100.

Parsons, K. C. (1995). International heat stress standards: a review. Ergonomics, 38 (1), 6-22.

Parsons, K. C. (2003). Human Thermal Environments (2nd ed.). London: Taylor and Francis.

Parsons, K. C. (2006). Heat stress standard ISO 7243 and its global application. Industrial Health, 44, 368-379.

Parsons, K. C., Havenith, G., Holmér, I., Nilsson, H., \& Malchaire, a. J. (1999). The effects of wind and human movement on the heat and vapour transfer properties of clothing. Annals of Occupational Hygiene, 43 (5), 347-352.

PMI. (2008). A Guide to the Project Management Body of Knowledge (PMBOK Guide, 4th Edition). Newtown Square, Pa.: Project Management Institute.

Ramphal-Naley, L. (2012). Screening for heat stress in workers and athletes. Baylor University Medical Center Proceedings, 25 (3), 224-228.

Rasmussen, J. (1997). Risk management in a dynamic society: a modelling problem. Safety Science, 27 (2/3), 183-213.

Raven, P. B., Drinkwater, B. L., Horvath, S. M., Ruhling, R. D., Gliner, J. A., Sutton, J. C., \& Bolduan, N. W. (1974). Age, smoking habits, heat stress, and their interavtive effects with Carbon Monoxide and Peroxyacetylinitrate on man's aerobic power. International Journal of Biometeorology, 18 (3), 222-232.

Rohles, F. H. (1973). The revised model comfort envelope. ASHRAE Transactions, 79 (II), 71-80.

Rowlinson, S. (1997). Hong Kong Construction Site Safety Management. Hong Kong: Sweet \& Maxwell Limited.

Saskatchewan Ministry of Labour and Workplace Safety. (2000). Working under Hot Conditions. Regina: Occupational Health and Safety Division, Saskatchewan Ministry of Labour and Workplace Safety, Canada.

Schulte, P., \& Chun, H. (2009). Climate change and occupational safety and health: establishing a preliminary framework. Journal of Occupational and Environmental Hygiene, 6, 542-554.

Senge, P. M. (2006). The fifth discipline : the art and practice of the learning organization (Rev. ed.). New York: Doubleday/Currency.

Sharma, V. M., Pichan, G., \& Panwar, M. R. (1983). Differential effects of hot-humid and hot-dry environments on mental functions. International Archives of Occupational and Environmental Health, 52, 315-327.

Steadman, R. G. (1979). The assessment of sultriness. Part I: A temperature-humidity index based on human physiology and clothing science. Journal of Applied Meterology, 18, 861-873. 
Steadman, R. G. (1984). A universal scale of apparent temperature. Journal of Climate and Applied Meteorology, 23, 1674-1687.

Stribley, R. F., \& Nunneley, S. A. (1978). Fighter Index of Thermal Stress: Development of Interim Guidance for Hot-Weather USAF Operations. Texas: US School of Aerospace Medicine SAM-TR-78-6.

Sutcliffe, K. M. (2011). High reliability organizations (HROs). Best Practice \& Research Clinical Anaesthesiology, 25 (2), 133-144.

Taylor, G., \& O'Sullivan, R. (2012). Risk management of heat exposure in mining. Safety Bulletin (Mines Inspectorate, Department of Employment, Economic Development and Innovation, Queensland Government, Australia), 115 (11 January 2012).

Tian, Z., Zhu, N., Zheng, G., \& Wei, H. (2011). Experimental study on physiological and psychological effects of heat acclimatization in extreme hot environments. Building and Environment, 46 (10), 2033-2041.

Vassallo, S. U., \& Delaney, K. A. (1989). Pharmacologic effects on thermoregulation: mechanisms of drugrelated heatstroke. Climnical Toxicology, 27 (485), 199-224.

Weick, E., \& Sutcliffe, K. M. (2007). Managing the Unexpected (2nd edition). San Francisco: Jossey-Bass.

Weick, K. E., Sutcliffe, K. M., \& Obstfeld, D. (1999). Organizing for high reliability: process of collective mindfulness. In R. S. Sutton \& B. M. Staw (Eds.), Research in Organizational Behavior, Volume 1 (pp. 81-123). Stanford: Jai Press.

WHO. (1969). Health Factors Involved in Working under Conditions of Heat Stress (World Health Organization Technical Report Series No. 412). Geneva: World Health Organization.

Work Safe Alberta. (2009). Best Practice - Working Safely in the Heat and Cold. Alberta: Government of Alberta, Employment and Immigration,.

Yaglou, C. P. (1950). awaiting for ILLiad. American Public Health Association Year Book 1949-1950, Part II, 40 (5), 131-143.

Yaglou, C. P., \& Minard, D. (1956). Prevention of Heat Causalities at Marine Corps Training Centers, Contract Report to ONR N5 ori-7665, May 31, 1956. Washington D. C.: Office of Naval Research, Physiology Branch.

Yaglou, C. P., \& Minard, D. (1957). Control of heat casualties at military training centers. American Medical Association Archives of Industrial Health, 16, 302-316.

YMCA of the USA. (2000). YMCA Fitness Testing and Assessment Manual (4th ed.). Champaign, Illinois: Human Kinetics.

Zhao, J., Zhu, N., \& Lu, S. (2009). Productivity model in hot and humid environment based on heat tolerance time analysis. Building and Environment, 44, 2202-2207.

Appendix A. Suggested screening items for personal risk factors and decisions on risk mitigation

\begin{tabular}{ll}
\hline Items & Suggested decision on mitigating the risk \\
\hline Gender & The difference mainly lies in habitual levels of exercise. Yet high level of \\
& heat stress risks endangering pregnancy. \\
Age & The process of aging is associated with brittle vasoregulatory \\
& mechanisms, declined fitness, development of chronicle illnesses and \\
& disabilities which may reduce sweating abilities and cardiovascular \\
& efficiency of distribute heat. \\
Cardiopulmonary fitness & Echocardiogram or electrocardiogram for heavy work; basic \\
& cardiovascular screening to workers above 35 years old engaging in \\
& moderate/heavy work. Other alternative methods include lung function \\
& testing, aerobic capacity test $\left(\mathrm{VO}_{2 \text { max }}\right)$, skinfold thickness test, etc.
\end{tabular}

Physical fitness Data can be obtained through YMCA step test (YMCA of the USA, 2000) or other step tests. Unfit workers should not be exposed to long period in the heat before they improve their fitness and cardiac conditioning.

Body temperature By internal abdominal temperature, rectal temperature, aural or tympanic temperature, oesophageal temperature, or peripheral skin temperature, for details see ISO 9886 (2004).

Obesity (height/weight) $\quad \mathrm{BMI}>30 \mathrm{~kg} / \mathrm{m}^{2}$ : requires health counseling about dietary \& exercise goals; $\mathrm{BMI}>40 \mathrm{~kg} / \mathrm{m}^{2}$ : work for very short periods of time with frequent break sessions until their BMI is lowered to a safer level.

Past history of heat illness Including history of heat stroke and inability to acclimatize, Indicate a reduced tolerance to heat stress. Two or more episodes imply the person is constantly intolerant to heat. 
History of other diseases

Temporary unfitness

Experience \& skills

Acclimatisation

High blood pressure

Diabetes

Impaired mental capacity,

Cardiac arrhythmias

Myocardial infarction

Cardiac stent placement

Cardiac bypass

Including skin disease such as allergy, chest disease such as asthma, syncopal attacks, epilepsy, or any other disease that affects the cardiovascular system, the sweating mechanism, the respiratory system or gastro-intestinal system or renal system that influence the fluid or electrolyte balance, skin disease

Individuals who report feeling unwell, e.g. vomiting or diarrhea, should not be exposed to heat. Individuals with any infect, dehydration, loss of sleep, skin trauma, or on low sodium diet need special care.

Inexperience of working in the heat or not skilled at specific job

Unacclimatised or newly return from illness or leave

Disqualify if blood pressure exceeds $160 / 100$

Urinalysis for everyone exposed to heat; Serum glucose testing for those urinalysis positive or demanded to do above medium level activities Serum glucose $>200 \mathrm{mg} / \mathrm{dL}$-stabilize the glucose before the individual is allowed into a hot environment

People with alzheimer's disease or Dementia or trisomy disorders should be put on mandatory work-rest cycles and remind to drink fluids

Disqualify if there is a malignant arrhythmia

Clear with benign arrhythmia after medication control and normal baseline stress echo biannually

Wait 2 months then clear with normal baseline stress echo and normal biannual echo; ejection fraction must be $>40 \%$

Wait 1 week them clear with normal baseline stress echo; repeat every 2 years

Wait 3 months then clear with normal baseline stress echo; repeat every 2 years; ejection fraction must be $>40 \%$

Appendix B Drugs and medication and their related health risks in heat (Ramphal-Naley, 2012; Vassallo \& Delaney, 1989)

\begin{tabular}{|c|c|}
\hline Items & Induced health risks in heat \\
\hline Alcohol & $\begin{array}{l}\text { Heart rate increase, dehydration, inducing inappropriate } \\
\text { behaviour, chronically abuse of alcohol impair the } \\
\text { thermoregulatory mechanism }\end{array}$ \\
\hline Smoking & $\begin{array}{l}\text { Increase blood pressure and heart rate, impair aerobic capacity } \\
\text { and lung ventilation }\end{array}$ \\
\hline Alpha adrenergic & Promote vasoconstriction and decrease skin blood flow \\
\hline Amphetamines & $\begin{array}{l}\text { Increase in heart rate and sweating, interfere with central thermal } \\
\text { regulation }\end{array}$ \\
\hline Anticholinergic & Increase in heart rate, limit sweating ability, especially for the \\
\hline Antihistamine & Increase in heart rate, abnormal sweating \\
\hline Antihypertensive & Slow down heart rate, low blood pressure \\
\hline Antipsychotics & Impair thermoregulation by acting on the central nervous system \\
\hline Barbiturates & Impair thermoregulation by acting on the central nervous system \\
\hline Benzodiazepine & Heart rate liability, abnormal sweating, impair thermoregulation \\
\hline Beta blockers & stabilize heart rate, decrease efficiency of transferring heat to skin \\
\hline Calcium channel blocker & Slow down heart rate, venous pooling, abnormal sweating \\
\hline Diuretics & Dehydration, decreased vasodilation \\
\hline Laxative & Heart rate liability, abnormal vasodilation \\
\hline $\begin{array}{l}\text { Lithium and haloperidol (used } \\
\text { together) }\end{array}$ & Elevate body temperature \\
\hline
\end{tabular}


Meperidine

Monoamine oxidase inhibitors

Neuroleptic

Phenothiazine

Sedatives

Thyroid agonist

Topiramate

Tricyclic antidepressant

Cocaine

Phencyclidine (PCP)

Lysergic acid diethylamide(LSD)
Elevate body temperature

Impair thermoregulation by acting on the central nervous system Heart rate liability, abnormal sweating

Electrolyte imbalance, prohibit sweating, deteriorate heat dissipation

Affect thirst thresholds

Increase in heart rate and sweating

Electrolyte imbalance

Electrolyte imbalance, Impair thermoregulation by acting on the central nervous system

Increase in heart rate and sweating, interfere with central control of thermal regulation

Abnormal temperature regulation

Increase in heart rate, abnormal sweating 